\title{
Two topics from lattice NRQCD at non-zero temperature: heavy quark mass dependence and S-wave bottomonium states moving in a thermal bath
}

\section{Seyong Kim*}

Department of Physics, Sejong University, Seoul 143-747, Korea

E-mail: skimesejong.ac.kr

\section{Gert Aarts, Chris Allton}

Department of Physics, Swansea University, Swansea, United Kingdom

\section{Maria Paola Lombardo}

INFN-Laboratori Nazionali di Frascati, I-00044, Frascati (RM), Italy

\section{Mehmet B. Oktay}

Physics Department, University of Utah, Salt Lake City, Utah, USA

\section{Sinead M. Ryan}

School of Mathematics, Trinity College, Dublin 2, Ireland

\section{Donald K. Sinclair}

HEP Division, Argonne National Laboratory, 9700 South Cass Avenue, Argonne, Ill 60439, USA

\section{Jon-Ivar Skullerud}

Department of Mathematical Physics, National University of Ireland Maynooth, Maynooth, Ireland

Using Non-Relativistic QCD (NRQCD), we study heavy quark mass dependence of S-wave and P-wave bottomonium correlators for $0.42 T_{c}<=T<=2.09 T_{c}$ and study spectral functions of Swave bottomonium states moving in a thermal bath at these temperatures using Maximum Entropy Method with NRQCD kernel. For the studied momentum range, the energy of moving states shows quadratic momentum-dependence and the width of moving states does not show significant changes as the momentum of bottomonium is increased. Also, we find that in correlator ratios, the temperature effect is larger than the effect caused by $20 \%$ change in the bottom quark mass.

The 30th International Symposium on Lattice Field Theory - Lattice 2012,

June 24-29, 2012

Cairns, Australia

${ }^{*}$ Speaker. 


\section{Introduction}

Quark-Gluon-Plasma (QGP) is a state of matter in the Strong Interaction system which is actively sought through theoretical study and heavy ion collision at high energy (or high temperature) and at low energy (or high baryon density) and must have played an important role in the evolution of early universe [1]. Unfortunately at physical quark masses, chiral symmetry restoration/deconfinement transition in QCD from normal hadronic matter to QGP matter is a cross-over transition and lacks characteristic behaviors of order parameter in a first order or a second order thermodynamic phase transition across the thermodynamic transition. Thus, finding and understanding physical phenomena which can serve as signatures for QGP matter formation is quite crucial.

Physics of heavy quarkonium in medium may be one of such candidate phenomena [2]. In contrast to large uncertainty in our understanding of light hadron production and decays, inclusive productions and decays of heavy quarkonium in proton-proton or proton-anti-proton collision, which are required for the comparison with those in heavy ion collisions, is well understood: Non-Relativistic QCD (NRQCD) factorization in which the scale above heavy quark (like charm quark or bottom quark) mass is "integrated away" and the smallness of heavy quark velocity at the quarkonium rest frame offers "small parameter" is applicable in heavy quarkonium [3]. Short distance effects can be calculated via perturbative expansion in $\alpha_{s}$ and long distance non-perturbative matrix elements can be computed either using lattice NRQCD at zero temperature $[4,5,6]$ or can be fitted using experimental data (see e.g., [7]). Based on our understanding of quarkonium physics in $\mathrm{p}-\mathrm{p}$ collision, medium effect for quarkonium in heavy ion collision may be better disentangled. In particular, bottomonium system offers a better probe for QGP formation since there are clearer seperations between the long distance physics and the short distance physics, compared to those in charmonium system due to smaller quark velocity $\left(v_{b}^{2} \sim 0.1\right.$ compared to $v_{c}^{2} \sim 0.3$ for charmonium system) at the quarkonium rest frame.

In recent years, we studied the behavior of S-wave and P-wave bottomonium states for a temperature range $0.4 T_{c} \leq T \leq 2.1 T_{c}$ using NRQCD in non-zero temperature on anisotropic lattices $\left(12^{3} \times N_{t}\right)$ with two light quark flavors [8,9]. Details on how dynamical gauge field is generated and what the simulation parameters are can be found in $[8,9,10]$. Our calculation method is explained in $[8,9]$. We expect that in-medium effect such as temperature $(T)$ effect or baryon chemical potential $(\mu)$ effect can be accommodated by NRQCD as long as $\frac{T}{M} \ll 1$ or $\frac{\mu}{M} \ll 1$ where $M$ is the heavy quark mass (see, e.g. [11] for baryon chemical potential efffect in $\mathrm{NRQC}_{2} \mathrm{D}$ ). Indeed our study $[8,9]$ meets such expectation. We found that P-wave bottomonium states $\left(\chi_{b J}\right)$ melt immediately above $T_{c}$, and bound state signals for S-wave channel $\left(\eta_{b}, \Upsilon\right)$ survive above $T_{c}$ [8]. Closer inspection of the spectral functions for S-wave correlators $\left(\chi_{b}, \Upsilon\right)$, which is obtained by the Maximum Entropy Method (MEM) with NRQCD kernel, shows that $(1 S)$ peaks appears to survive and the excited state peaks are suppressed [9]. This observation is consistent with recent CMS experiment which shows sequential suppression of $(3 S),(2 S) \Upsilon$ states and survival of $(1 S) \Upsilon$ $[12,13]$.

Here, we discuss further lattice NRQCD study of ours on bottomonium at non-zero temperature. First, bottomonium with non-zero momentum is studied (for full report, see [14]). Quarkonium production in hadron collision proceeds: parton evolution from hadron, then partonic scat- 

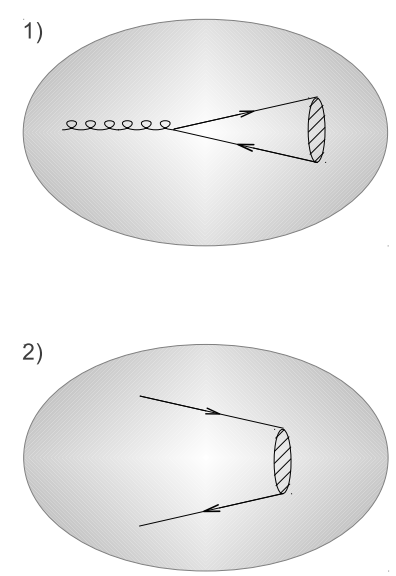

Figure 1: Quarkonium production processes: (a)fragmentation from gluon (top), (b) recombination of thermalized heavy quark-heavy anti-quark (bottom)

tering process (gluon fusion, heavy quark recombination, gluon Compton scattering, and light quark-light quark scattering). Among these partonic processes, quarkonium production is mostly dominated by gluon fusion. Gluon subsequently fragments into quarkonium. In this case, fragmenting gluon will have large energy and momentum. If quarkonium is produced in thermal bath, then it will be moving relative to thermal bath. In contrast, if quarkonium is produced through recombination of thermalized heavy quark and heavy anti-quark (albeit less probable), produced quarkonium is at rest in thermal bath. Schematic view on these two processes is shown in Fig. 1. Since a thermal bath defines a preferred rest frame, "moving" effect will induce $\frac{1}{2} M v^{2}$ shift in the mass compared to the mass of quarkonium produced at rest. This shift may be larger than experimental resolution for muon detection and may be observable.

Secondly, we start to investigate systematic errors associated with our computation in $[8,9]$. As a first step, we investigate how $10 \sim 20 \%$ change in bottom quark mass affects previous observations made in [8,9] by calculating bottomonium correlators with $M a_{s}=3.7,4.14$ and 5.4 and looking at the correlator ratios.

\section{S-wave bottomonium moving in thermal bath}

We consider $\eta_{b}$ and $\Upsilon$ which are moving with regard to the thermal bath, i.e., S-wave bottomonium states with momentum. Non-zero momentum quarkonium state is obtained by injecting momentum to the source for NRQCD quark propagator computation and projecting out the injected momentum component from the quarkonium propagator which is obtained by combining the zero-momentum NRQCD quark propagator with the momentum injected NRQCD quark propagator. Momenta, $\vec{k}=\frac{2 \pi}{L}\left(n_{1}, n_{2}, n_{3}\right)$ where $\vec{n}=(0,0,0),(1,0,0),(1,1,0),(1,1,1),(2,0,0),(2,1,0)$, and $(2,2,0)$ with $L=12$, have been considered. Since $M a_{s}=4.5$ is used for the bottom quark mass in NRQCD propagator computation and $M_{\eta(1 S)}=9.391 \mathrm{GeV}$ and $M_{\Upsilon(1 S)}=9.460 \mathrm{GeV}$ [15], these quarkonium momentum range amount to quarkonium velocity, $0.07 \lesssim v \lesssim 0.18$ with 


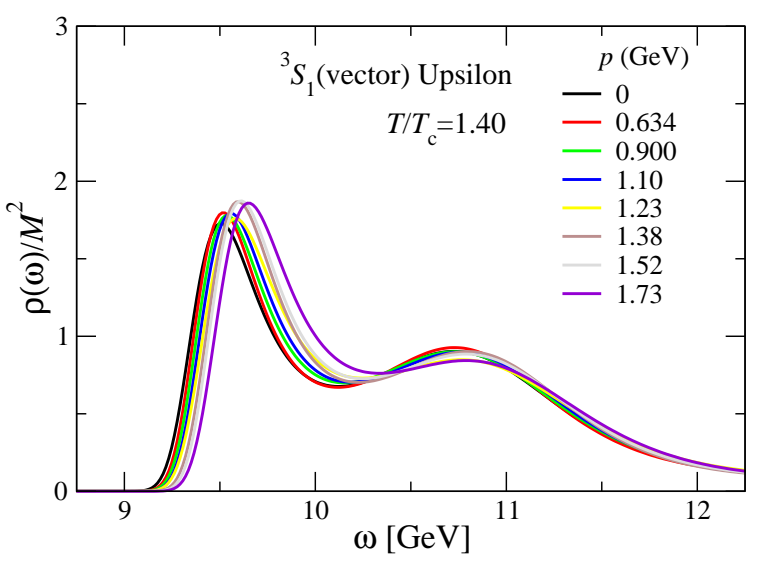

Figure 2: $\Upsilon$ spectral functions on $12^{3} \times 24$ for various momenta

$\vec{p}^{2} a_{s}^{2}=4 \sum_{i=1,3} \sin ^{2} \frac{k_{i}}{2}$. From the correlators, spectral functions defined as

$$
G(\tau, \vec{p})=\int \frac{d \omega}{2 \pi} K(\tau, \omega) \rho(\omega, \vec{p}), \quad K(\tau, \omega)=e^{-\omega \tau}
$$

are computed using MEM. Fig. 2 shows a typical spectral function as a function of $|\vec{p}|$. For each momentum, the spectral function exhibits a distinct shape. From this, the peak and width can be extracted and Fig. 3 show extracted peak values and width values which corresponds the $\Upsilon(1 S)$ state. To a leading order in $\vec{p}^{2}$ and $T$, we see that the $1 S$ peak roughly follows

$$
\Delta E(\vec{p}, T) \simeq \Delta E(\vec{p}=0, T=0)+\frac{\vec{p}}{2 M}+c\left(\alpha_{S}\right) T
$$

In general, continuum NRQCD dispersion is

$$
E=M_{0}+\frac{\vec{p}^{2}}{2 M_{2}}-\frac{\left(\vec{p}^{2}\right)}{8 M_{4}^{3}}+\cdots
$$

Since we use $\mathscr{O}\left(v^{4}\right)$ NRQCD lagrangian to compute bottomonium propagator, $M_{0}$ has $\mathscr{O}\left(v^{4}\right)$ error, $M_{2}$ has $\mathscr{O}\left(v^{2}\right)$ error, and $M_{4} \mathscr{O}\left(v^{0}\right)$ respectively. Using the form of $M_{0}+4 \sum_{i}\left(\sin \left(k_{i} / 2\right)^{2}\right) / 2 M_{2}-$ $\left(4 \sum_{i}\left(\sin \left(k_{i} / 2\right)^{2}\right)^{2}\right) / 8 M_{2}^{3}$ to fit $N_{t}=80 \Upsilon$ correlator, we get $11.05 \mathrm{GeV}$ for the kinetic mass $\left(M_{2}\right)$ of $\Upsilon(1 S)$ state with $M a_{s}=4.5$. The fitting form $M_{0}+4 \sum_{i}\left(\sin \left(k_{i} / 2\right)^{2}\right) / 2 M_{2}$ gives $11.12 \mathrm{GeV}$ for $M_{2}$. Thus Eq. (2.2) is suitable to describe our lattice result. On the other hand, the $1 S$ width is independent of $\vec{p}^{2}$ and is just proportional to $T$.

This lattice NRQCD finding can be compared with effective theory prediction. NRQCD bound state at rest in thermal bath below the melting temperature has been considered in [16] and nonrelativistic bound state which is moving with regard to thermal bath has been considered in QED (in the rest frame of the bound state, i.e., bound state in moving thermal bath) [17]. Finding in [17] for the width can be summarized as

$$
\frac{\Gamma_{v}}{\Gamma_{0}}=\frac{\sqrt{1-v^{2}}}{2 v} \log \left(\frac{1+v}{1-v}\right) \rightarrow 1-\frac{2}{3} v^{2}+\mathscr{O}\left(v^{4}\right)
$$



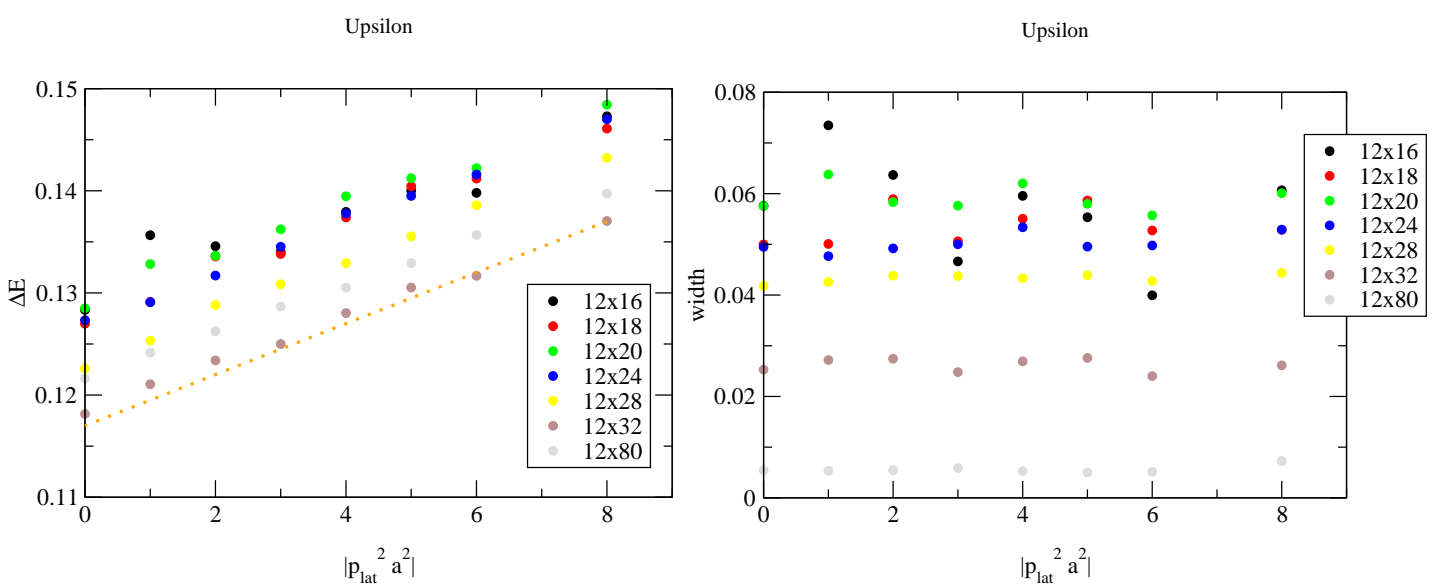

Figure 3: $\Upsilon(1 S)$ energy vs. various momenta from the first peak of the spectral functions at each $T / T_{c}=$ $2.09,1.86,1.68,1.40,1.20,1.05$, and 0.42 for $N_{t}=16,18,20,24,28,32,80$ respectively (left). $\Upsilon(1 S)$ width vs. various momenta from the width of the first peak in spectral functions (right)

where $\Gamma_{0}$ is the width of the bound state not moving with regard to thermal bath and $\Gamma_{v}$ is the width of the bound state moving with the velocity $v$ in thermal bath. With $v^{2}<0.04$, "moving effect" in the width is beyond the precision level of our computation, which explains negligible momentum dependence of the width in the right figure of Fig. 3.

\section{Mass dependence of bottomonium correlators}

Our previous bottomonium study was done with bottom quark mass, $M a_{s}=4.5$. The same NRQCD correlator calculation is repeated with $M a_{s}=3.7(-20 \%), 4.14(-10 \%)$ and $5.4(+20 \%)$ in order to investigate how small change in bottom quark mass affects bottomonium spectrum and how important this effect is compared to non-zero temperature effect. Since lattice NRQCD is an effective theory and is consistent when $M a_{\tau} \sim 1$, all these choices $\left(M a_{\tau}=0.62,0.75,0.9\right.$ for $M a_{s}=3,7,4.5,5.4$ respectively) are expected to be valid.

Lattice NRQCD quarkonium correlator behaves as $A e^{-\Delta E \tau}$ when a bound state is formed and fitting $\Upsilon$ correlator calculated on $N_{t}=80$ configurations gives $\Delta E_{\Upsilon}(M, T \sim 0)$. Using experimental value for $M_{\Upsilon}$, we determine the unknown constant for NRQCD spectrum by

$$
M_{\Upsilon}^{\text {exp }}=E_{0}(M)+\Delta E_{\Upsilon}(M, T=0)
$$

If non-zero temperature quarkonium states has a pole like $\Upsilon(1 S)$ state [9],

$$
M_{\Upsilon}(T)=E_{0}+\Delta E_{\Upsilon}(M, T)=M_{\Upsilon}^{\exp }+\Delta E_{\Upsilon}(M, T)-\Delta E_{\Upsilon}(M, T=0) .
$$

So, the ratio of the non-zero temperature quarkonium correlator to the $N_{t}=80$ correlator can reveal the heavy quark mass dependence $\Delta E(M, T)$ although the true ground state behaviors for $N_{t}=80$ correlators and for $N_{t}=16$ or 20 correlators will not set in at large $\tau$. Fig. 4 shows such ratios for $T=458\left(N_{t}=16\right)(\mathrm{MeV})$ and $T=408\left(N_{t}=18\right)(\mathrm{MeV})$. 


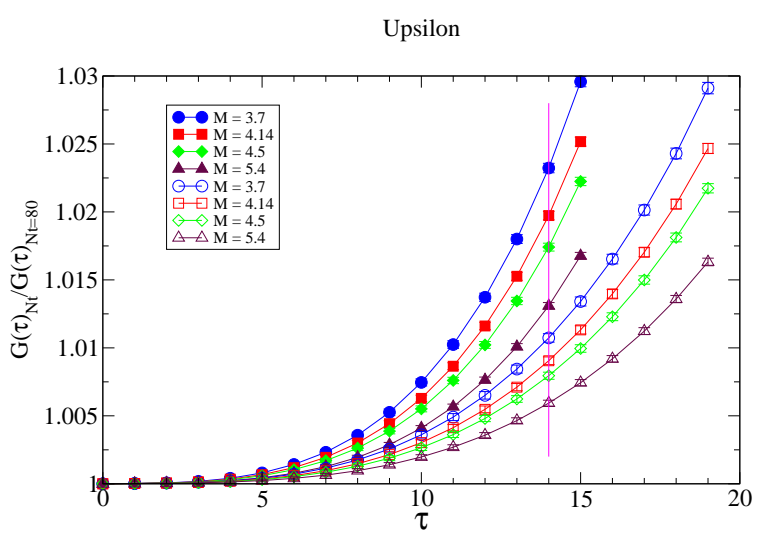

Figure 4: $\Upsilon$ correlator ratio of $G\left(\tau ; N_{t}\right)$ to $G\left(\tau ; N_{t}=80\right)$ for $N_{t}=16$ (filled symbol) and 18 (open symbol)

In Fig. 4, the comparison between the filled symbol and the empty symbol shows the temperature dependence for the same $M$, and the comparison between the different symbols shows $M$-dependence for the same $T$. The correlator ratio change due to $10 \%$ change in the two different $T$ is larger than due to $10 \sim 20 \%$ change in the 4 different $M$. Since the small change in heavy quark mass is less important than that due to the temperature change, lattice NRQCD is valid as an effective theory and systematic error due to heavy quark mass mis-tuning is small.

\section{Conclusion}

Using NRQCD formalism in non-zero temperature, we computed momentum dependent $\left(\eta_{b}, \Upsilon\right)$ correlators and calculated spectral functions using MEM via NRQCD kernel. From the peaks and the width of momentum dependent spectral function, we find that there are observable $v^{2}$ effect on the energy of S-wave state moving in thermal bath but no observable effect on the width of S-wave state moving in thermal bath for $v_{\text {upsilon }}^{2} \lesssim 0.04$.

Among various sources of systematic errors involved in our result, bottomonium correlators calculation is repeated with $20 \%$ variation in bottom quark mass. By studying correlator ratios, We find that the temperature effect is more important than the heavy quark mass effect in S-wave bottomonium at the temperature around a few $T_{c}$, which suggests lattice NRQCD as an effective theory for bottomonium in non-zero temperature is a consistent theory.

Improved tuning for bottom quark mass and detecting thermal deviations from the standard dispersion relation is in progress for $N_{f}=2+1$ flavor system with smaller spatial lattice spacing and a larger spatial extent.

\section{Acknowledgements}

We acknowledge the support and infrastructure provided by the Trinity Centre for High Performance Computing and the IITAC project funded by the HEA under the Program for Research in Third Level Institutes (PRTLI) co-funded by the Irish Government and the European Union. The work of CA and GA is carried as part of the UKQCD collaboration and the DiRAC Facility jointly 
funded by STFC, the Large Facilities Capital Fund of BIS and Swansea University. GA and CA are supported by STFC. SK is grateful to STFC for a Visiting Researcher Grant and INFN for the visit to Frascati, and is supported by the National Research Foundation of Korea grant funded by the Korea government (MEST) No. 2011-0026688. SR is supported by the Research Executive Agency (REA) of the European Union under Grant Agreement number PITN-GA-2009-238353 (ITN STRONGnet) and the Science Foundation Ireland, grant no. 11-RFP.1-PHY-3201. DKS is supported in part by US Department of Energy contract DE-AC02-06CH11357. JIS has been supported by Science Foundation Ireland grant 08-RFP-PHY1462 and 11-RFP.1-PHY-3193-STTF11.

\section{References}

[1] K. Yagi, T. Hatsuda and Y. Miake, "Quark-gluon plasma: From big bang to little bang”, Camb. Monogr. Part. Phys. Nucl. Phys. Cosmol. 23 (2005) 1.

[2] T. Matsui and H. Satz, Phys. Lett. B 178 (1986) 416.

[3] G. T. Bodwin, E. Braaten and G. P. Lepage, Phys. Rev. D 51 (1995) 1125 [Erratum-ibid. D 55 (1997) 5853] [arXiv:hep-ph/9407339].

[4] G. T. Bodwin, D. K. Sinclair and S. Kim, Phys. Rev. Lett. 77 (1996) 2376 [hep-lat/9605023].

[5] G. T. Bodwin, D. K. Sinclair and S. Kim, Phys. Rev. D 65 (2002) 054504 [hep-lat/0107011].

[6] G. T. Bodwin, J. Lee and D. K. Sinclair, Phys. Rev. D 72 (2005) 014009 [hep-lat/0503032].

[7] M. Butenschoen and B. A. Kniehl, Phys. Rev. Lett. 106 (2011) 022003 [arXiv:1009.5662 [hep-ph]].

[8] G. Aarts, S. Kim, M. P. Lombardo, M. B. Oktay, S. M. Ryan, D. K. Sinclair and J. -I. Skullerud, Phys. Rev. Lett. 106 (2011) 061602 [arXiv:1010.3725 [hep-lat]].

[9] G. Aarts, C. Allton, S. Kim, M. P. Lombardo, M. B. Oktay, S. M. Ryan, D. K. Sinclair and J. I. Skullerud, JHEP 1111 (2011) 103 [arXiv:1109.4496 [hep-lat]].

[10] R. Morrin, A. O. Cais, M. Peardon, S. M. Ryan and J. I. Skullerud, Phys. Rev. D 74 (2006) 014505 [arXiv:hep-lat/0604021].

[11] S. Hands, S. Kim and J. -I. Skullerud, Phys. Lett. B 711 (2012) 199 [arXiv:1202.4353 [hep-lat]].

[12] S. Chatrchyan et al. [CMS Collaboration], Phys. Rev. Lett. 107 (2011) 052302 [arXiv:1105.4894 [nucl-ex]].

[13] S. Chatrchyan et al. [CMS Collaboration], arXiv:1208.2826 [nucl-ex].

[14] G. Aarts, C. Allton, S. Kim, M. P. Lombardo, M. B. Oktay, S. M. Ryan, D. K. Sinclair and J. -I. Skullerud, arXiv:1210.2903 [hep-lat].

[15] J. Beringer et al. [Particle Data Group Collaboration], Phys. Rev. D 86 (2012) 010001.

[16] N. Brambilla, M. A. Escobedo, J. Ghiglieri, J. Soto, A. Vairo, JHEP 1009 (2010) 038 [arXiv:1007.4156 [hep-ph]].

[17] M. A. Escobedo, J. Soto and M. Mannarelli, Phys. Rev. D 84 (2011) 016008 [arXiv:1105.1249 [hep-ph]].

[18] F. Karsch, M. T. Mehr and H. Satz, Z. Phys. C 37 (1988) 617. 